\title{
Which anesthesia for carotid endarterectomy: Local or general?
}

\author{
Eyupserhat Calik, Umit Arslan and Bilgehan Erkut* \\ Department of Cardiovascular Surgery, Atatürk University, Medical Faculty, Turkey
}

Atherosclerotic disease of the carotid artery is a pathologically vital that may cause transient ischemic attacks, cerebral hypoperfusion, and syncope. Removal of the stenotic lesion with open surgical (carotid endarterectomy) or stent is the primary treatment modality. Carotid endarterectomy with general anesthesia has recently been performed by local anesthesia in many clinics. Despite limited work, complication rates were less common in carotid endarterectomy with local anesthesia. In this Editorial, we compare the results of our patients with local and general anesthesia.

We performed carotid endarterectomy in 26 patients. Twelve had local anesthesia (G1) and 14 had general anesthesia (G2). Patients did not have additional cardiac and coronary artery surgery. The carotid artery disease was diagnosed by carotid magnetic resonance imaging angiography in most patients (Figure 1). All of the patients were performed patch-plasty with pericardial patch and shunt was not used (Figure 2). There was no difference between patients in terms of cross clamping and operation time. In G1, trans-ischemic attack was observed in 1 patient and consciousness blurred in 2 patients per-operatively. The cross clamp was removed, and the operation was continued with vessel hangers. The vascular hangers were loosened intermittently to allow cerebral perfusion. Although there is a certain amount of bleeding, operations completed. In G2, trans-ischemic attack in 2 patients and stroke in 2 patients were detected, postoperatively. Patients were not coma but had right hemiplegia. No cardiac complications such as

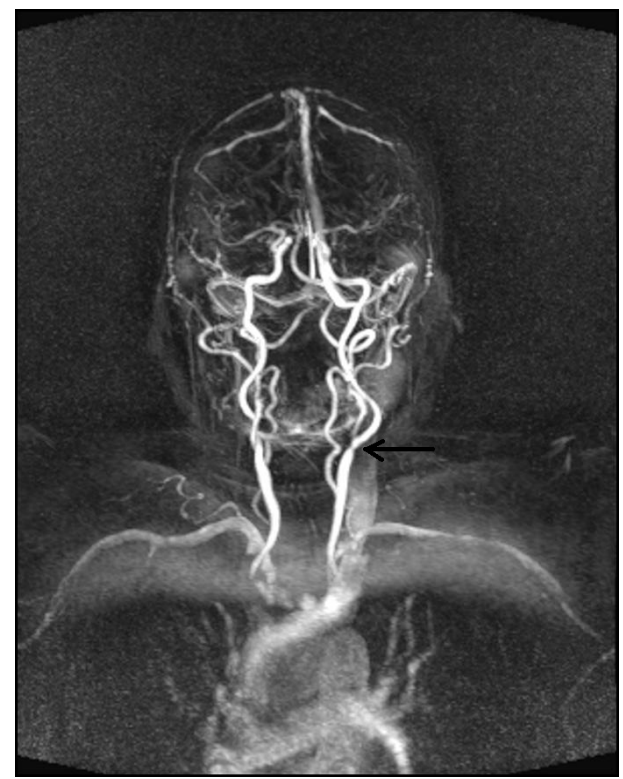

Figure 1. MR angiography shows carotid artery stenosis in a patient (black arrow)

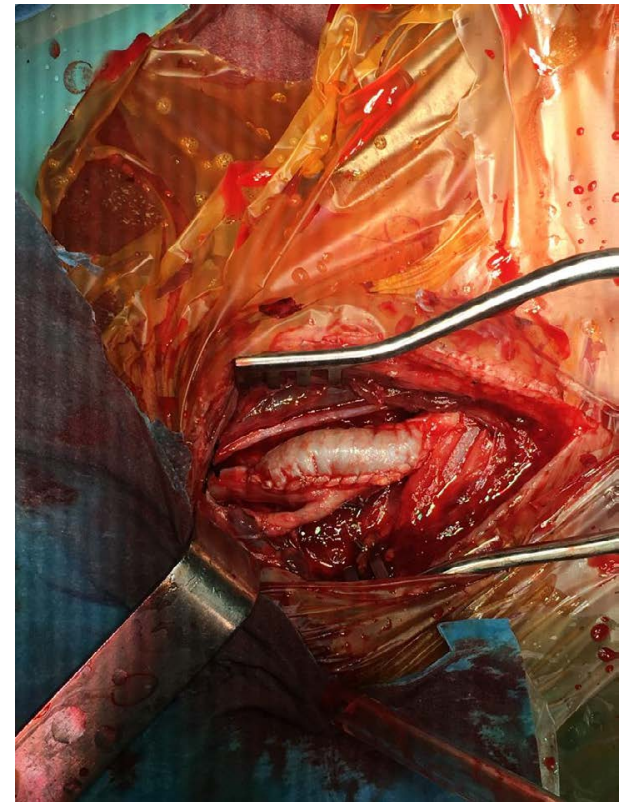

Figure 2. Per-operative view in a patient who underwent patch-plasty after endarterectomy

myocardial infarction and mortality were observed in both groups of patients. In two patients who underwent general anesthesia and developed hemiplegia, postoperative pneumonia developed and was treated. The duration of hospital stay in group G2 was longer than in G1, and G2 was higher when hospital costs were compared.

The roots of C2, C3 and C4 spinal nerves are blocked by local anesthesia for carotid endarterectomy. Ultrasound-guided blockage provides anesthesia both the superficial skin and the behind of sternocleidomastoid muscle $[1,2]$. Cerebral evaluation of the patient with the carotid artery clamp during the surgical procedure with general anesthesia is difficult. The clamping of the internal carotid artery may cause feeding problems in the willis polligon and its branches. This leads to cerebral ischemia and neurological damage. The cerebral oxygenation can be monitored with trans-cranial Doppler or

${ }^{\star}$ Correspondence to: Bilgehan Erkut, Atatürk University Medical Faculty, Department of Cardiovascular Surgery, Erzurum, Turkey, E-mail: bilgehanerkut@yahoo.com

Key words: carotid atherosclerosis, endarterectomy, local anesthesia, general anesthesia

Received: May 28, 2018; Accepted: July 27, 2018; Published: August 02, 2018 
near-infrared spectroscopy [3]. However, these methods are not a good indicator of the protection of cerebral blood flow. When used locally instead of general anesthesia, cerebral nutrition, assessment of blood flow and presence of neurological damage can be performed before the patient is awakened. During local anesthesia; handshake, visual and auditory stimulus responses such as awake tests can be performed, and the cerebral disturbance can be evaluated instantaneously. The identification of neurological pathologies and evaluation of cerebral functions is impossible during general anesthesia.

The mortality was not influenced by the type of anesthesia used for carotid surgery. But, difference was detected in terms of the neurological complications, hospital stay and hospital costs between general and local anesthesia. We believe that carotid endarterectomy should be performed with local anesthesia, although further studies or clinical trials are needed.

\section{Declaration of conflicting interests}

The author(s) declared no conflicts of interest with respect to the authorship and/or publication of this article.

\section{Funding}

The author(s) received no financial support for the research and/or authorship of this article.

\section{References}

1. Martusevicius R, Swiatek F, Joergensen LG, Nielsen HB (2012) Ultrasoundguided locoregional anaesthesia for carotid endarterectomy: a prospective observational study. Eur J Vasc Endovasc Surg 44: 27-30. [Crossref]

2. Cedergreen P, Swiatek F and Nielsen HB (2016) Local anaesthesia for carotid endarterectomy Pro: protect the brain. Eur J Anaesthesiol 33: 236-237. [Crossref]

3. Nielsen HB (2014) Systematic review of near-infrared spectroscopy determined cerebral oxygenation during noncardiac surgery. Front Physiol 5: 93. [Crossref]

Copyright: (C2018 Calik E. This is an open-access article distributed under the terms of the Creative Commons Attribution License, which permits unrestricted use, distribution, and reproduction in any medium, provided the original author and source are credited. 